\title{
AHLFORS FUNCTIONS ON DENJOY DOMAINS
}

\author{
AKIRA YAMADA \\ (Communicated by Clifford J. Earle, Jr.)
}

\begin{abstract}
Let $\Delta$ be the open unit disc. We give a characterization of a set that is the complement in $\Delta$ of the image of the Ahlfors function for some maximal Denjoy domain and $\infty$. As a corollary, we show by an example that there exists such a set with positive logarithmic capacity.
\end{abstract}

\section{INTRODUCTION}

Let $\Omega$ be a region on the Riemann sphere $\widehat{\mathbb{C}}$ that supports nonconstant bounded analytic functions and let $p \in \Omega$. Set $\mathbf{B}=\{f \mid f$ is holomorphic in $\Omega$ and $f(\Omega) \subset \Delta\}$ where $\Delta$ is the unit disc $\{z|| z \mid<1\}$. The Ahlfors function for $\Omega$ and $p$ is the unique function $F$ [1] in $B$ such that

$$
F^{\prime}(p)=\max _{f \in \mathbf{B}} \operatorname{Re} f^{\prime}(p) .
$$

By linear transformation, we see easily that $F(p)=0$. It is well known [6; 1, Theorem 3] that the image $F(\Omega)$ of any Ahlfors function $F$ covers the unit disc with the exception of a set of analytic capacity zero. We are interested in a characterization of this exceptional set that is called the omitted set of $F$. In this respect we restrict ourselves to study only Ahlfors functions whose domains of definition are maximal regions for bounded analytic functions in the sense of Rudin [10], since otherwise the above problem becomes less interesting [7]. Some examples of omitted sets of Ahlfors functions were given by several authors. Roding [9] gave an example of an omitted set consisting of two points. Minda [7] extended this example to fairly general discrete sets, and the author [13] gave examples of fairly general sets of logarithmic capacity zero.

As a first step toward the characterization of the omitted set we study the simplest case where the domain $\Omega$ is a Denjoy domain defined as follows: A planar domain $D \ni \infty$ is called a Denjoy domain if $\partial D$ is a compact subset of the real axis $\mathbb{R}$. The beautiful idea of using a Denjoy domain to study the omitted set of Ahlfors function is due to Minda [7]. The main result of our paper gives a necessary and sufficient condition for a subset of the unit disc to be the omitted set of the Ahlfors function $F$ for some maximal Denjoy domain and

Received by the editors October 24, 1990.

1991 Mathematics Subject Classification. Primary 30D50; Secondary 30C85. main.

Key words and phrases. Ahlfors function, analytic capacity, logarithmic capacity, Denjoy do- 
$\infty$ such that $F$ is a covering onto its image. As a corollary we give examples of omitted sets of Ahlfors functions that have positive logarithmic capacity. In [2] Fisher raised a question whether the composite function $F \circ \phi$ is an inner function on $\Delta$ where $F$ is the Ahlfors function for a maximal domain $D$ and $\phi: \Delta \rightarrow D$ is its uniformizer. Our examples immediately give another proof of a negative answer to this question, which was first shown by Gamelin [3, p. 93].

\section{Some Lemmas}

Let $\Omega$ be a Denjoy domain with boundary $E \subset \mathbb{R}$ that supports nonconstant bounded analytic functions. The following well-known formula [8] gives a useful integral representation of the Ahlfors function.

Lemma 1 (Pommerenke). The Ahlfors function $F$ for a Denjoy domain $\Omega$ and $\infty$ is given by

$$
F(z)=\tanh \left(\frac{1}{4} \int_{E} \frac{d \zeta}{z-\zeta}\right), \quad z \in \Omega .
$$

From Lemma 1, we obtain another representation of $F$ by using harmonic measure.

Lemma 2. The Ahlfors function $F$ for a Denjoy domain $\Omega$ and $\infty$ is given by

$$
F(z)=-i \tan \left(\frac{\pi}{4}\left[\omega_{E}(z)+i \omega_{E}^{*}(z)\right]\right), \quad z \in \Omega,
$$

where $\omega_{E}(z)=\frac{1}{\pi} \int_{E} \operatorname{Im}(\zeta-z)^{-1} d \zeta, z \in \Omega$, is the harmonic measure of $E$ relative to $H$, the upper half plane, and $\omega_{E}^{*}$ its harmonic conjugate function.

Proof. Recall that the Poisson integral formula for a bounded harmonic function $f$ on $H$ is given by

$$
f(z)=\frac{1}{\pi} \int_{-\infty}^{\infty} \tilde{f}(\zeta) \operatorname{Im} \frac{1}{\zeta-z} d \zeta
$$

where $\tilde{f}$ is the nontangential limit of $f$. Thus,

$$
\begin{aligned}
\frac{1}{4} \int_{E} \frac{d \zeta}{z-\zeta} & =-\frac{1}{4} \int_{E}\left(\operatorname{Re} \frac{1}{\zeta-z}+i \operatorname{Im} \frac{1}{\zeta-z}\right) d \zeta \\
& =-\frac{\pi i}{4}\left(\omega_{E}(z)+i \omega_{E}^{*}(z)\right) .
\end{aligned}
$$

In view of Lemma 1 , this gives the lemma.

Lemma 3. Let $F$ be the Ahlfors function for a Denjoy domain $\Omega$ and $\infty$. Then $F$ satisfies the identity $F(\bar{z})=\overline{F(z)}$ for all $z \in \Omega$. Moreover, $\operatorname{Im} F(z)<0$ if and only if $z \in \Omega \cap H$.

Proof. From Lemma 1 it is clear that the above identity holds. By Lemma 2, we have

A calculation shows that

$$
\operatorname{Im} F(z)=-\operatorname{Re} \tan \left(\frac{\pi}{4}\left(\omega_{E}+i \omega_{E}^{*}\right)\right) .
$$

$$
\operatorname{Im} F(z)=-\tan \frac{\pi}{4} \omega_{E} /\left(\cosh ^{2} \frac{\pi}{4} \omega_{E}^{*}+\sinh ^{2} \frac{\pi}{4} \omega_{E}^{*} \tan ^{2} \frac{\pi}{4} \omega_{E}\right) .
$$

Since $F$ is nonconstant, the linear measure of the boundary $E$ is positive. Thus the harmonic measure $\omega_{E}$ satisfies the inequality $0<\omega_{E}<1$ on $H$. This trivially implies the second statement of the lemma. 


\section{Denjoy domain of TyPe $(K, \pi)$}

Given a set $X \subset \mathbb{C}$ let us denote by $\mathrm{Cl}(X)$ its closure and by $\bar{X}$ the reflection of the set $X$ in the real axis. Let $\Sigma$ be the family of relatively closed subsets of $\Delta$ satisfying:

(1) $K$ has analytic capacity zero,

(2) $K=\bar{K}$,

(3) $K \cap \mathbb{R}=\mathrm{Cl}(K \backslash \mathbb{R}) \cap \mathbb{R}$,

(4) $0 \notin K$.

The following construction of a maximal Denjoy domain is a slight extension of the one introduced by Minda [7]. For any $K \in \Sigma$ let $\pi: H \rightarrow \Delta_{-} \backslash K$ be a holomorphic universal covering with the cover transformation group $\Gamma$ where $\Delta_{-}=\Delta \cap \bar{H}$ denotes the lower half disc. Let $A$ be the set of points $p$ in $\partial H$ such that the covering $\pi$ has a continuous extension to some neighborhood $(\subset \partial H)$ of $p$ where $\pi$ takes real boundary values everywhere. Since conditions (1) and (3) imply that $\Delta_{-} \backslash K$ has a free boundary arc, we see that $\Gamma$ is a Fuchsian group of the second kind acting on $H$ and that $A$ is a nonempty open subset of $\partial H$. Replacing $\pi$ with $\pi \circ \gamma$ for some $\gamma \in \operatorname{Möb}(H)$, the Möbius transformation group acting on $H$, if necessary, we assume here and hereafter that $\infty \in A$ and that $\pi(\infty)=0$. This is possible by condition (4). By Schwarz reflection principle the covering $\pi$ is continued holomorphically to the Denjoy domain $\Omega=H \cup A \cup \bar{H}$. We use the same notation $\pi$ to denote the extended map. Since by condition (3) each component of the set $(-1,1) \backslash K$ is a free boundary arc of $\Delta_{-} \backslash K$, we have an important observation that $A$ is a $\Gamma$-invariant subset of $\partial H$ and $\pi(A)=(-1,1) \backslash K$. One verifies easily that the map $\pi: \Omega \rightarrow \Delta \backslash K$ is a holomorphic covering of $\Delta \backslash K$ with the cover transformation group $\Gamma$. Note that since $\pi$ maps $H$ to $\bar{H}$ and $\pi(\infty)=0$, we have $\pi^{\prime}(\infty)=\lim _{z \rightarrow \infty} z(\pi(z)-\pi(\infty))>0$. The domain $\Omega$ constructed above is called the Denjoy domain of type $(K, \pi)$. We remark that the covering $\pi$ is a nonconstant bounded analytic function on $\Omega$.

Wee need four lemmas.

Lemma 4. Let $\Omega$ be the Denjoy domain of type $(K, \pi)$. Then $\Omega$ is simply connected if and only if $K=\varnothing$.

Proof. We assume that $\Omega$ is simply connected, since if $K=\varnothing$ the lemma is clear. Then $\Omega$ is of the form $\widehat{\mathbb{C}} \backslash[a, b]$ for some $a$ and $b \in \mathbb{R}(a<b)$. since the interval $[a, b]$ is $\Gamma$-invariant, it is easy to see that each $\gamma \in \Gamma$ fixes $a$ and $b$ where $\Gamma$ is the cover transformation group of the covering $\pi$. It follows from discontinuity of $\Gamma$ that the group $\Gamma$ is either trivial or hyperbolic cyclic. Since $\Delta \backslash K$ is conformally equivalent to the quotient surface $\Omega / \Gamma$, we have a bounded univalent function $f: \Delta \backslash K \rightarrow S$ where $S$ is either $\Delta$ or an annulus. Since $K$ has analytic capacity zero, $K$ is a removable set for bounded analytic functions $[4$, p. 10]. Hence $f$ is extended to a univalent function on $\Delta$ whose image is the same $S$. This implies that $K=\varnothing$.

Lemma 5. The Denjoy domain of type $(K, \pi)$ is maximal.

Proof. By definition the covering $\pi$ satisfies the identity $\pi(\bar{z})=\overline{\pi(z)}, z \in \Omega$. Assume that $\pi$ has an analytic extension to some neighborhood $U$ of a point $p \in \mathbb{R}$. Then the above identity implies that $\pi(U \cap \mathbb{R}) \subset \mathbb{R}$. By definition of 
the set $\Omega \cap \mathbb{R}$, we have $p \in \Omega \cap \mathbb{R} \subset \Omega$. Since $\pi$ is a bounded analytic function on $\Omega$, this implies that any point of $\partial \Omega$ is an essential boundary point.

Lemma 6. Let $\Omega$ be a maximal Denjoy domain that supports nonconstant bounded analytic functions, and let $F$ be the Ahlfors function for $\Omega$ and $\infty$ with the omitted set $K$. If $F$ is a covering onto its image, then $K \in \Sigma$ and $\Omega$ is the Denjoy domain of type $(K, F)$.

Proof. By Lemma 3, the restriction $\left.F\right|_{H}$ is a universal covering onto the domain $\Delta_{-} \backslash K$. Set $K^{\prime}=\mathrm{Cl}(K \backslash \mathbb{R}) \cap \Delta \quad(\subset K)$. We claim that $K^{\prime} \in \Sigma$. By definition, conditions (2) and (3) defining the family $\Sigma$ are trivially satisfied, and (4) is clear since $P(\infty)=0$. Since the image of an Ahlfors function is the unit disc with the exception of a set with analytic capacity zero [1], condition (1) also holds. Hence the claim is proved.

Because the restriction $\left.F\right|_{H}$ is a universal covering of $\Delta_{-} \backslash K^{\prime}$, let $D$ be the Denjoy domain of type $\left(K^{\prime},\left.F\right|_{H}\right)$. To conclude the proof, we must show that $D=\Omega$ and $K^{\prime}=K$. Since $F(\Omega \cap \mathbb{R}) \subset(-1,1)$ by Lemma 3, recalling the definition of the set $D \cap \mathbb{R}$, we obtain $\Omega \subset D$. Assume that there exists a point $p \in D \backslash \Omega$. Since the domain $\Omega$ is maximal, $p$ is an essential boundary point of $\Omega$. Then again the definition of the set $D \cap \mathbb{R}$ shows that $F$ has a holomorphic extension to some neighborhood of $p$ and that $F(p) \in(-1,1)$. This, however, contradicts the fact that if $p$ is an essential boundary point of $\Omega$, then by [2, Corollary] $\lim \sup _{x \rightarrow p}|F(x)|=1$. Hence $D=\Omega$ and we have $F(\Omega)=\Delta \backslash K^{\prime}$, so $K^{\prime}=K$ as desired.

Lemma 7. Let $\Omega$ be the Denjoy domain of type $(K, \pi)$, and let $f$ be the Ahlfors function for $\Omega$ and $\infty$. If the image $f(\Omega)$ is a proper subset of $\Delta$, then we have $f=\pi$ and $f(\Omega)=\Delta \backslash K$.

Proof. Let $\Gamma$ be the cover transformation group of the covering $\pi$. From Lemma 1 , using a fact that $\partial \Omega$ is $\Gamma$-invariant, a direct calculation shows that for $\gamma \in \Gamma, f \circ \gamma=\phi(\gamma) \circ f$, where $\phi(\gamma)$ is a hyperbolic element with fixed points at \pm 1 of the form $\tau^{-1} \circ A_{\gamma} \circ \tau, A_{\gamma}(z)=e^{M(\gamma)} z, M(\gamma) \in \mathbb{R}$, and $\tau(z)=(1+z) /(1-z)$. Indeed, from Lemma 1 we see by calculation that the map $M: \Gamma \rightarrow \mathbb{R}$ is a group homomorphism, that is, $M(\gamma \circ \delta)=M(\gamma)+M(\delta)$ for any $\gamma$ and $\delta \in \Gamma$.

First, we claim that the subgroup $M(\Gamma) \subset \mathbb{R}$ is discrete. Note that, since $\phi(\gamma)$ is a conformal automorphism of the image $f(\Omega), \phi(\gamma)$ is also a bijective self-map of $\Delta \backslash f(\Omega) \quad(\neq \varnothing)$. If $M(\Gamma)$ is not discrete, then it is elementary to see that $M(\Gamma)$ is dense in $\mathbb{R}$. Considering the points $\phi(\gamma)(p)$ for all $\gamma \in \Gamma$ with some fixed point $p \in \Delta \backslash f(\Omega)$, we find that the set $\Delta \backslash f(\Omega)$ contains a dense subset of a circular arc with its end points at 1 and -1 . Since $\Delta \backslash f(\Omega)$ is closed, $\Delta \backslash f(\Omega)$ contains a circular arc. This contradicts the fact that the analytic capacity of the set $\Delta \backslash f(\Omega)$ is zero. Thus, $M(\Gamma)$ is discrete and the claim is proved.

Again it is elementary to se that if $M(\Gamma)$ is discrete then $M(\Gamma)$ is cyclic. Hence the subgroup $\phi(\Gamma) \subset \operatorname{Möb}(\Delta)$ is a cyclic group $\langle\alpha\rangle$ with generator $\alpha \epsilon$ $\operatorname{Möb}(\Delta)$. We claim that $f$ is $\Gamma$-invariant. If $\alpha$ is the identity, then $\phi(\Gamma)$ is a trivial group and we have nothing to prove. Thus we may assume that $\alpha$ is hyperbolic and that the quotient surface $\Delta /\langle\alpha\rangle$ is an annulus. Let $p: \Delta \rightarrow \Delta /\langle\alpha\rangle$ be the natural projection. Because $p \circ f: \Omega \rightarrow \Delta /\langle\alpha\rangle$ is $\Gamma$-invariant, the map 
$p \circ f \circ \pi^{-1}: \Delta \backslash K \rightarrow \Delta /\langle\alpha\rangle$ is a well-defined bounded analytic function. Since $K$ has analytic capacity zero, $p \circ f \circ \pi^{-1}$ is extended to a holomorphic function $g: \Delta \rightarrow \Delta /\langle\alpha\rangle$. Then since the map $p$ is a universal covering, there exists a lifting $h: \Delta \rightarrow \Delta$ of $g$ such that $g=p \circ h$. Thus, $p \circ f=p \circ h \circ \pi$ on $\Omega$. Hence we have $f=\varepsilon \circ h \circ \pi$ for some $\varepsilon \in\langle\alpha\rangle$, showing that $f$ is $\Gamma$-invariant and the claim is proved.

Since $f$ is $\Gamma$-invariant, $f \circ \pi^{-1}: \Delta \backslash K \rightarrow \Delta$ is a well-defined bounded analytic function and hence is extended to a holomorphic function $\rho: \Delta \rightarrow \Delta$. Because $f=\rho \circ \pi$ and $f(\infty)=\pi(\infty)=0$, we have $\rho(0)=0$ and $f^{\prime}(\infty)=\rho^{\prime}(0) \pi^{\prime}(\infty)$. By extremality of the Ahlfors function, we have $\rho^{\prime}(0) \geq 1$ since $f^{\prime}(\infty)>0$ and $\pi^{\prime}(\infty)>0$. Then Schwarz lemma implies that $\rho(z)=z$ and hence $f=\pi$.

\section{MAin Results}

Theorem 1. Let $\Omega$ be the Denjoy domain of type $(K, \pi)$, and let $\Gamma$ be the cover transformation group of the covering $\pi$. If $f$ is the Ahlfors function for $\Omega$ and $\infty$, then the following are equivalent.

(1) $f$ is $\Gamma$-invariant, i.e., $f \circ \gamma=f$ for all $\gamma \in \Gamma$;

(2) $f=\pi$;

(3) $f$ is a covering onto its image;

(4) $f(\Omega)=\Delta \backslash K$;

(5) $K \backslash \mathbb{R}$ has logarithmic capacity zero.

Moreover, if $\Omega$ is not simply connected, then each of the above conditions is equivalent to the following.

(6) $f(\Omega) \neq \Delta$.

Proof. First we show that (1) implies (2). If (1) holds, then the same reasoning as in the last part of the proof of Lemma 7 shows that $f=\pi$, and we see that condition (2) holds.

That (2) implies (3) is trivial.

Next assume that (3) holds. If $f(\Omega)=\Delta$ and $f$ is a covering, then since $\Delta$ is simply connected, $\Omega$ is also simply connected. Lemma 4 implies that $K=\varnothing$, and hence (4) holds. On the other hand, if $f(\Omega)$ is a proper subset of $\Delta$, then Lemma 7 shows that (4) holds. Hence (3) implies (4).

Assume that (4) holds. To prove (5) we may assume that $K \neq \varnothing$. Then from Lemma 7 condition (4) implies that $f=\pi$ and so $f$ is $\Gamma$-invariant. We show that if $f$ is $\Gamma$-invariant then (5) holds. Since $E=\partial \Omega$ is $\Gamma$-invariant, one verifies easily that the harmonic measure $\omega_{E}$ is $\Gamma$-invariant. From Lemma 2 we see that the conjugate harmonic function $\omega_{E}^{*}$ is also $\Gamma$-invariant. Since $g=\omega_{E}+i \omega_{E}^{*}$ is $\Gamma$-invariant, there exists a holomorphic function $h$ defined on $\Delta \backslash K$ such that $g=h \circ \pi$ with $0<\operatorname{Re} h<1$ on $\Delta_{-} \backslash K$. Since by definition the analytic capacity of $K$ is zero, considering the bounded analytic function $(h-1) /(h+1)$, we may assume by analytic continuation that $h$ is holomorphic on $\Delta_{-}$. Set $\omega_{0}=\operatorname{Re} h$. Then $\omega_{0}$ is harmonic on $\Delta_{-}$and satisfies an identity $\omega_{E}=\omega_{0} \circ \pi$. By definition the nontangential boundary value of $\left.\omega_{E}\right|_{H}$ is 0 or 1 a.e. on the real axis. On the other hand, it follows from maximum principle for harmonic functions that $0<\omega_{0}<1$ for all $z \in \Delta_{-}$. Hence we find that almost all nontangential boundary values of $\left.\pi\right|_{H}$ belong to $\partial \Delta_{-}$. It is elementary to construct a conformal mapping $k: \Delta_{-} \rightarrow \Delta$. Then we conclude that $k \circ \pi: H \rightarrow$ 
$\Delta$ is an inner function; i.e., the absolute value of its nontangential boundary values is a.e. equal to one. Frostman's theorem [5, p. 79] implies that the image of any inner function covers the unit disk with the exception of a set of logarithmic capacity zero. Thus the set $k\left(K \cap \Delta_{-}\right)$has logarithmic capacity zero. This in turn implies by the conformal invariance of vanishing of logarithmic capacity [11, p. 184] that $K \cap \Delta_{-}$also has logarithmic capacity zero. Since $K \backslash \mathbb{R}=\left(K \cap \Delta_{-}\right) \cup \overline{\left(K \cap \Delta_{-}\right)}$, by using a fact that a countable union of sets of logarithmic capacity zero is of logarithmic capacity zero [12, p. 57], we see that (5) holds.

We proceed to show that if $K \cap \Delta_{-}$has logarithmic capacity zero then $f$ is $\Gamma$-invariant. In view of Lemma 2 , it suffices to show that the conjugate harmonic function $\omega_{E}^{*}$ is $\Gamma$-invariant since $\omega_{E}$ is $\Gamma$-invariant. As before set $\omega_{E}=\omega_{0} \circ \pi$ where $\omega_{0}$ is a bounded harmonic function on $\Delta_{-} \backslash K$. The assumption implies that $\omega_{0}$ is extended to a bounded harmonic function on $\Delta_{-}[12$, p. 78]. Since any $\Gamma$-period of $\omega_{E}^{*}$ is a flux of $\omega_{0}$ along some closed curve in $\Delta_{-} \backslash K$, all periods must vanish. Thus, $\omega_{E}^{*}$ is $\Gamma$-invariant.

Finally, the last assertion is clear from Lemmas 4 and 7 . This completes the proof of Theorem 1.

Corollary 1. Let $K$ be a subset of $\Delta$. Then the following are equivalent.

(1) $K$ is the omitted set of the Ahlfors function $F$ for some maximal Denjoy domain and $\infty$ that is covering onto its image;

(2) $K \in \Sigma$ and $K \backslash \mathbb{R}$ has logarithmic capacity zero.

Proof. If condition (1) holds, then by Lemma 6 the domain of definition of $F$ is a Denjoy domain $\Omega$ of type $\left(K^{\prime}, \pi\right)$ for some $K^{\prime} \in \Sigma$ and $\pi$. Theorem 1 then implies that $f(\Omega)=\Delta \backslash K^{\prime}$. Thus $K=K^{\prime}$. Hence by Theorem 1 the set $K$ satisfies (2).

Conversely, if $K$ satisfies (2), let $\Omega$ be the Denjoy domain of type $(K, \pi)$ for some covering $\pi$. Again, Theorem 1 shows that if $K \backslash \mathbb{R}$ has logarithmic capacity zero, then $K$ is the omitted set of the Ahlfors function $\pi$ for $\Omega$ and $\infty$.

Corollary 2. There exists a maximal Denjoy domain $\Omega$ such that the omitted set of the Ahlfors function for $\Omega$ and $\infty$ has positive logarithmic capacity.

Proof. Let. $S \subset(0,1)$ be a compact set with zero linear measure but with positive logarithmic capacity. Using the Cantor ternary set on $[0,1]$, we easily obtain such a set. Take a countable dense subset $\left\{a_{n}\right\}_{n=1}^{\infty}$ of $S$. Let

$$
K=S \cup\left\{z \mid z=a_{n} \pm i / k(n, k \in \mathbb{N}, k \geq n) \text { and }|z|<1\right\} .
$$

Then it is clear that $K \in \Sigma$ and that $K \backslash \mathbb{R}$ has zero logarithmic capacity. Corollary 1 implies that $K$ is an omitted set with positive logarithmic capacity.

Let $f$ be a bounded analytic function on a hyperbolic domain $D$. Then $f$ is called an inner function if and only if $f \circ \phi$ is a usual inner function on $\Delta$ where $\phi: \Delta \rightarrow D$ denotes a holomorphic universal covering of $D$. Frostman's theorem [5, p. 79] implies that if a function is inner then its omitted set has logarithmic capacity zero. Corollary 2 immediately gives the following result.

Corollary 3. There exists a maximal Denjoy domain $\Omega$ such that the Ahlfors function for $\Omega$ and $\infty$ is not an inner function. 


\section{REFERENCES}

1. S. D. Fisher, On Schwarz's lemma and inner functions, Trans. Amer. Math. Soc. 138 (1969), 229-240.

2. _ The moduli of extremal functions, Michigan Math. J. 19 (1972), 179-183.

3. T. Gamelin, Lectures on $H^{\infty}(D)$, Notas Mat., La Plata, Argentina, Vol. 21, 1972.

4. J. B. Garnett, Analytic capacity and measure, Springer-Verlag, Berlin, Heidelberg, and New York, 1972.

5. __ Bounded analytic functions, Academic Press, New York, 1981.

6. S. Ya. Havinson, Analytic capacity of sets, joint nontriviality of various classes of analytic functions and the Schwarz lemma in arbitrary domains, Amer. Math. Soc. Transl. Ser. 2, vol. 43, Amer. Math. Soc., Providence, RI, 1964, pp. 215-266.

7. C. D. Minda, The image of the Ahlfors function, Proc. Amer. Math. Soc. 83 (1981), 751-756.

8. Ch. Pommerenke, Über die analytische Kapazität, Arch. Math. (Basel) 11 (1960), 270-277.

9. E. Roding, Über die Wertannahme der Ahlfors funktion in beliebigen Gebieten, Manuscripta Math. 20 (1977), 133-140.

10. W. Rudin, Some theorems on bounded analytic functions, Trans. Amer. Math. Soc. 78 (1955), 333-342.

11. L. Sario and K. Oikawa, Capacity functions, Grundlehren Math. Wiss., vol. 149, SpringerVerlag, Berlin, Heidelberg, and New York, 1969.

12. M. Tsuji, Potential theory in modern function theory, Maruzen, Tokyo, 1959.

13. A. Yamada, A remark on the image of the Ahlfors function, Proc. Amer. Math. Soc. 88 (1983), 639-642.

Department of Mathematics, Tokyo Gakugei University, Nukuikita-Machi, KoganeiSHI, TOKYO 184, JAPAN

E-mail address: yamada@clezio.u-gakugei.ac.jp 